\title{
Comparative transcription analysis of hard and tender fruit spines of cucumber to identify genes involved in morphological development of fruit spines
}

\section{Duo Lv}

Shanghai Jiao Tong University

Yao Yu

Shanghai Jiao Tong University

Liang-Rong Xiong

Shanghai Jiao Tong University

\section{Gang Wang}

Shanghai Jiao Tong University

Jin-An Pang

Tianjin diret seeds company limited

Jing-Xian Sun

Shanghai Jiao Tong University

Ke-Yan Zhang

Shanghai Jiao Tong University

Huan-Le He

Shanghai Jiao Tong University

Run Cai

Shanghai Jiao Tong University

Junsong Pan ( $\boldsymbol{\nabla}$ jspan71@sjtu.edu.cn )

Shanghai Jiao Tong University https://orcid.org/0000-0003-3983-1285

Research article

Keywords: Cucumber, Trichomes, Fruit spine, Transcriptome analysis, Morphological development

Posted Date: June 8th, 2020

DOI: https://doi.org/10.21203/rs.3.rs-31353/v1

License: (c) (1) This work is licensed under a Creative Commons Attribution 4.0 International License. Read Full License 
Page 2/31 


\section{Abstract \\ Background}

The trichomes of cucumber on the fruit are also called spines, it not only has important commercial value but also is a classical tissue to study cell division and differentiation mode of multicellular trichomes. Although there have been many researches about the development of unicellular trichomes in model plants, the molecular mechanism of multicellular trichomes formation remains elusive. In this study, we took a pair of cucumber materials defined as hard (Ts, wild-type) and tender spines (ts, mutant) in previous study, the whole developmental process of fruit spines was continuously observed by microscope and SEM, in an attempt to define the development stage of fruit spines, transcriptome profiles at different stages was made to explore the molecular mechanism in the process of spines development.

\section{Results}

With significant phenotypic differences, the developmental process of fruit spines was clearly defined as four stages. Comparion of transcriptome profilings showed a total of 2,788 differential expression genes (DEGs) between the wild-type and mutant at different developmental stages of fruit spines, these genes exhibited different expression patterns at different spines developmental stage. Some DEGs related to cell cycle and meristem had also been identified in transcriptome data. By correlating the expression patterns of several transcription factors that have been reported to be involved in the development of spines, we identified some genes that may be involved in the formation of cucumber fruit spines. The cluster analysis of transcription factors revealed that there were 26 transcription factor families that may be involved in the development of fruit spines, among them, the ERF-ERF, bHLH and WAKY family transcription factor gene families were the top three gene families.

\section{Conclusions}

We defined the four stages of fruit spines development and identified a number of genes that may be involved in the development of multicellular trichomes based on comparative transcription. The results provided a step stone for further analysis of the molecular mechanism in the developmental process of multicellular trichomes.

\section{Background}

Trichomes are hairy structure covering the epidermis of plant. As one tissue that contacts with environment, they play an important role in protecting plant against pathogens, insects, UV irradiation, and reducing loss of heat and moisture. (Hülskamp et al., 1998; Hülskamp, 2004; Schellmann and Hülskamp, 2005). Based on Morphological characteristics, trichomes can be divided into several forms: 
unicellular or multicellular, glandular or glandless, and branched or unbranched (Werker 2000). So trichomes provide an excellent model for studying cell differentiation and proliferation (Hülskamp 2004; Szymanski et al. 2000).

Arabidopsis is a classical model plant, the studies about its trichomes are wide and deep. Trichomes of Arabidopsis are single cell structure, they originate from epidermis cells and are widely distributed on leaves, stems, and sepals (Pesch and Hülskamp, 2004). The development process of trichomes is generally divided into six stages. A directional epidermal cell (precursor) expands rapidly relative to the surrounding epidermal cell, when precursor expands to 2-3 fold larger than surrounding cells, its surface will appear stalks. Stalks gradually develop into branches, and then the cell size continues to increase. After that, the top of branches becomes sharp, which means the maturation of trichome. Finally, a papillate surface will around the mature trichomes (Szymanski et al., 1998; Johnson et al., 2002). The cell differentiation of trichomes in Arabidopsis is regulated by a series of competing transcription factors (Zhao et al., 2015b). GLABRA1 (GL1) and MYB23 are both R2R3-MYB (v-myb avian myeloblastosis viral oncogene homolog) transcription factors, which can activate the initiation of trichomes (Oppenheimer et al., 1991). They are functionally redundant and specifically expressed in the early developmental trichomes. WD-repeat protein TRANSPARENT TESTA GLABRA1 (TTG1) can interact with GL1 and affects the development of trichomes, the mutant of TTG1 will exhibit a complete absence of trichomes (Galway et al., 1994; Walker et al., 1999). Two basic helix-loop-helix (bHLH) proteins GLABRA3 (GL3) and ENHANCER OF GLABRA3 (EGL3) can positively regulate the density and cell structure of trichomes (Payne et al., 2000; Zhang et al., 2003). There are also some negative factors involved in trichome developmental process. Single-repeat MYB proteins TRIPTYCHON (TRY) (Schellmann S et al., 2002; Pesch and Hülskamp, 2011), CAPRICE (CPC) (Wada et al., 2002), ENHANCER OF TRY AND CPC1 (ETC1), ETC2, and CAPRICE-LIKE MYB3 (Esch et al., 2004; Kirik et al., 2004a, b; Simon et al., 2007) act partially redundantly as negative regulators, they can inhibit the activity of the complex (MYB-WD40-bHLH) by competing with the R2R3 MYB protein to bind the bHLH protein (Kirik et al. 2004b; Wester et al. 2009).

Cucumber is an annual species that is commercially important worldwide (Zhao et al., 2015a). According to the previous study (Chen et al., 2014), unlike the trichomes of Arabidopsis, cucumber has glandular and non-glandular multicellular trichomes at the same time. The non-glandular multicellular trichomes are visible throughout the above-ground part of the cucumber plant, especially the trichomes on the fruit, also known as the fruit spines, it is also an important agronomic traits that affects commercial value. Comparing with trichomes of other tissues, the structural differentiation of the fruit spines is more obvious. The basic structure of fruit spines can be divided into a plinth of many cells and a stalk of single cells joined together. Until now, some key genes about development of cucumber fruit spines have been identified. CsTril/ CsGL3 (trichome-less) (Pan et al., 2015; Wang et al., 2015) is a class IV homeodomainleucine zipper (HD-Zip IV) transcription factor, it can activate the initiation of trichomes. The plant of mutant Cstril is glabrous. CsMict/ CsGL1/ TBH (Micro-trichome) is a member of the class I homeodomain-leucine zipper (HD-Zip IV) family, and it can affect the number and development of cucumber trichomes (Chen et al., 2014; Li et al., 2015; Zhao et al., 2015b). The results of genetic analysis and transcriptome profiling indicated that the CsTril had an epistatic effect on the CsMict in trichome 
development (Pan et al., 2015; Wang et al., 2015). CsMict also can interact with CsTTG1, a homologous protein of TTG1, to affect the number and size of the spines in the cucumber (Chen et al., 2016). One $\mathrm{C} 2 \mathrm{H} 2$ zinc finger transcription factor CsTu (tuberculate fruit) can affect the development of tubercules that under the fruit spines by regulating CTK biosynthesis (Yang et al., 2014). CsTs (tender spines), a Ctype Lectin receptor-like kinase (LecRLK), can affect the structure of trichomes. The mutantions of CsTs will cause the cells from basic part of trichomes were arranged out-of-order from the normal style of wildtype (Wt) (Guo et al., 2017), but cannot affect the density and size of trichomes, which indicates that CsTs is a key gene determining the differentiation of cucumber trichomes. To the best of our knowledge, there is no detailed characterization about the development regulation of cucumber trichomes. In this study, we compared and explored the developmental process of cucumber trichomes in the wild-type and a mutant of CsTs, tender spines (Csts). We further performed comparative transcriptome profiling analyses to identify genes and gene networks that might involve in cucumber trichome development.

\section{Results}

\section{Developmental stages of cucumber fruit trichomes}

The trichomes are widely distributed on the leaves, tendrils, stems, calyx and ovary of cucumber. Trichomes could be divided into two types. Type I is generally visible to the naked eye, it has obvious spiny structure (Fig.1A). The characteristics of type I trichomes could be specifically described as a pyramidal apical cell at the top, it connected to a cylindrical structure consisting of $5 \sim 8$ single cells, forming the stalk of trichomes. Below the stalk was a base made of hundreds of spherical cells. The structure of type I trichomes on different tissues was generally similar, but type I trichomes on the cucumber ovary was larger in size and stronger in hardness, so it is called spines. Contrary to type I, type II trichomes were much smaller, their top was composed of four cells that were not very distinct from each other, this top was connected to the epidermis by $3 \sim 5$ cells (Fig.1B).

Since spines were more consistent on ovary than other type I trichomes on other tissues in the development state, and cells had higher hardness and larger size, which was convenient for making plant slices, so we decided to take spines as the research object to explore the development process of type I trichomes. Combined with previous studies, with the help of microscope and SEM observation, the development process of spines could be divided into four stages (Fig. 2A-I).

In stage I, there would be pus-like protrusions on the cucumber epidermis where the spines would develop, and then the apical cells would develop first in these protrusions; in stage II (Fig. 2A, D, G), the development of stalk was initiated. The mode of development was that the single cells that made up stalk develop in the form of a relay, when one cell began to develop, the next cell was flat like a cake. This 'cake-like' cell would not begin to lengthen until the last cell complete developed. This stage continued until the ovary grew to approximately $0.25 \sim 0.30 \mathrm{~cm}$ in length, and stalk of spines would have four complete cells. During stage I and stage II, the ovary was relatively thin, and because of the low rigidity of the newly developed spines, they would cling to the ovary like "fetal hair", while the population of cells 
that later develop as bases also underwent a modest elongation, but would not have a complete initiation of development. At start of stage III (Fig. 2B, E, H), the ovary swelled obviously and grew to $0.35 \mathrm{~cm}$ in length. The fifth single cell completes develops, the rigidity of the spines begins to increase, causing the stalk to become erect. This stage would continue until the ovary length was about $0.65 \mathrm{~cm}$. The end of stage III also meant the end of stalk development. The mature stalk was composed 7 8 fully elongated single cells. The morphology of stalk was top narrow bottom wide. The cells that develop later were larger than the one that grew before, but the cell connected to the base (the last single cell) would be shorter than other single cells to ensure structural stability. In stage IV (Fig. 2C, F, I), the population of cells that made up the base began to multiply and expand the volume of cells until development completed, forming a spherical pedestal.

\section{CsTs influences the development of spines after stage II}

In order to determine the specific stages which caused the difference of spines form between wild-type (CsTs) and mutant (Csts), we continuously compared the spines development state on different stages of ovary.

In stage I and II (Fig. 3A, D, G), the spines development state of mutant was consistent with wild-type, they both had four complete single cells which cling to the ovary like "fetal hair". When in stage III (Fig. 3B, E, $\mathrm{H})$, started with the fifth cell, all single cells that made up stalk would not have a fully elongated like wildtype, they were stacked together like a multi-layer pie. At the same time, the growth and division mode of single cells (after fifth single cell) also changed, some got shorter and some divided into multiple cells. In the mutant, stage IV (Fig. 3C, F, I) no longer seemed to exist, and the boundary between base and stalk was no longer obvious. Base of spines was no longer a spherical structure, but an oval structure that linked a shorter stalk and epidermis of cucumber. The narrow lower part of the oval structure would lead to the unstable binding of spines on the epidermis, it resulted in the spines of mutant appeared to be soft and elastic. Finally, the mature spines would be distorted due to the disorder of the cell division mode, the shape of spines would be curved, and a large vacuole would form in the fifth single cell. From stage III, although the stalk (or spines) would be erected due to the rigidity of the stalk began to increase, the insufficient development of cell would lead to a disorganized arrangement of spines on the mutant ovary

surface compare to wild type. We also observed hairs on receptacles of wild-type and mutant, and found that they also had this structural difference (Fig. 4). However, no difference in root hair structure was found between wild type and mutant by either microscope or SEM (Fig. 5).

\section{Transcriptome analyses of cucumber fruit spines}

In order to exploring the gene network in different stages of spines development between wild-type and mutant, we collected their spines from three different developmental stages (stage II, stage III and stage IV) for RNA-seq, respectively. The RNA-seq was performed for three biological replicates in each stage. On average, about $5.94 \mathrm{~Gb}$ bases were generated from each sample by Illumina Hiseq platform. After mapping sequenced reads to reference genome (Cucumber 9930 v2 Genome), a total of 23,943 genes were obtained from all samples, including 695 new genes. The data quality of RNA-Seq summary is 
shown in Table 1. We also calculated the correlation between the samples from different stages and materials, to test if the samples chosen were reliable. The result showed that the three replicates at three stages showed a good correlation (Fig. 6).

Using the thresholds of false discovery rate $\leq 0.05$, absolute Log 2 Ratio value $\geq 1.5$, we divided the transcriptome data into three groups for analysis (Table S2). Group I, we compared FPKM values between different libraries of wild-type spines at three developmental stages, they were Wt-stagelll vs Wt-stagell, Wt-stagelV vs Wt-stagell, and Wt-stagelll vs Wt-stagell. 1,090 DEGs were found from stage II to stage III (510 up-regulated, 580 down-regulated), 2,594 DEGs were found from stage II to stage IV (1,481 upregulated, 1,113 down-regulated), 1,719 DEGs were found from stage III to stage IV (1,030 up-regulated, 689 down-regulated) (Fig. 7A-C). Group II, we compared FPKM values between different libraries of mutant spines at each two adjacent developmental stages, they were Mu-stagelll vs Mu-stagell MustagelV vs Mu-stagell, and Mu-stagelll vs Mu-stagell, 703 DEGs were found were found from stage II to stage III (294 up-regulated, 409 down-regulated), 1,929 DEGs were found from stage II to stage IV (853 upregulated, 1,076 down-regulated), 1,064 DEGs were found from stage III to stage IV (475 up-upregulated, 589 down-regulated) (Fig. 7A-C). Group III, we compared FPKM values between different libraries of wildtype and mutant spines at each same developmental stage, they were Wt-stagell vs Mu-stagell, Wtstagelll vs Mu-stagelll, and Wt-stagelV vs Mu-stagelV. 1,747 DEGs were found at Wt-stagell vs Mu-stagell (1,094 up-regulated, 653 down-regulated), 1,537 DEGs at Wt-stagell vs Mu-stagelll (814 up-regulated, 723 down-regulated), 1,166 DEGs were found at Wt-stagelV vs Mu-stagelV (507 up-regulated, 659 downregulated) (Fig. 7D). In total, there were 3,248 and 2,327 DEGs were involved in the three stages of spines development in the wild-type and mutant respectively. In total of 2,788 DEGs were found between the wild-type and mutant.

\section{GO and KEGG analysis of the DEGs}

GO and KEGG are the two most commonly useful programs for annotation and functional categorization of genes. The DEGs from wild-type, mutant and between wild-type and mutant in three developmental stages of spines were aligned to GO and KEGG databases.

In the wild-type (3248 DEGs), there were 1446, 2046, and 1742 DEGs were assigned to the molecular function, the biological process, and the cellular component categories through the $\mathrm{GO}$ analysis, respectively (Fig. 8A). The largest two subcategories in each of the 'biological process', the 'cellular component', and the 'molecular function' categories were 'metabolic process' (1386 DEGs) and 'singleorganism process' (1376 DEGs), the 'cell part' (1530 DEGs) and 'cell' (1517 DEGs), and the 'catalytic activity' (1296 DEGs) and 'binding' (1096 DEGs), respectively. Based on the comparison against the KEGG database, 580 DEGs were assigned to 112 pathways (Table S3). The largest three pathways were Plant hormone signal transduction (64 DEGs), Starch and sucrose metabolism (45 DEGs) and Carbon metabolism (43 DEGs). 
In the mutant (2327 DEGs), the result of GO analysis showed that 1389, 1478, and 1214 DEGs were assigned to the molecular function, the biological process, and the cellular component categories, respectively (Fig. 8B). The largest two subcategories in each of the 'biological process', the 'cellular component', and the 'molecular function' categories were 'metabolic process' (1010 DEGs) and 'singleorganism process' (967 DEGs), the 'cell part' (1055 DEGs) and 'cell' (1048 DEGs), and the 'catalytic activity' (982 DEGs) and 'binding' (774 DEGs), respectively. 439 DEGs were assigned to 106 pathways (Table S4). The largest three pathways were Phenylpropanoid biosynthesis (47 DEGs), Plant hormone signal transduction (44 DEGs) and Starch and sucrose metabolism (33 DEGs).

Between wild-type and mutant, A total of 2327 DEGs were assigned to different GO ontologies based on their sequence similarity to the genes with previously known functions; specifically, 1643, 1776, and 1451 DEGs were assigned to the molecular function, the biological process, and the cellular component categories, respectively (Fig. 8C). The largest two subcategories in each of the 'biological process', the 'cellular component', and the'molecular function' categories were 'metabolic process' (1197 DEGs) and 'cellular process' (1146 DEGs), the 'cell part' (1264 DEGs) and 'cell' (1254 DEGs), and the 'catalytic activity' (1128 DEGs) and 'binding' (924 DEGs), respectively. 519 DEGs were assigned to 105 pathways (Table S5). The largest three pathways were Plant hormone signal transduction (54 DEGs), Phenylpropanoid biosynthesis (52 DEGs) and Starch and sucrose metabolism (42 DEGs).

\section{The DEGs related to Meristem and Cell Cycle}

Through phenotypic observation, the cell division pattern was the mainly reason that caused the differences between wild-type and mutant, at the same time, previous researches also reported that genes involve in meristem regulation may play an important role in the development of spines (Chen et al., 2014). So we analyzed transcriptome data to explore their expression characteristics. We identified 78 genes involved in meristem regulation from DEGs (MRDs) between wild-type and mutant. There were 51, 38 and 26 MRDs in three stages respectively. The analysis result implied that MRDs in different development stage of spines had different regulation mode of (Table S6-S8). Eight genes, included several genes well-known to be involved in the development of plant tissues, showed expression level difference in all three stages between wild-type and mutant. For example, Csa3G824990 is a NAC transcription factor, its expression was down-regulated by 1.7, 1.9 and 4.1 fold in each stage of the mutant, the homologous gene of Csa3G824990 can be regulated by microRNA and is widely involved in the regulatory network of leaf shaping and development of lateral root in Arabidopsis (Patrick at al., 2004; Nikovics et al., 2006). The expression level of another NAC transcription factor (Csa1G575100) was also up-regulated in in wild-type, its expression was up regulated by 1.9, 2.8 and 2.3 fold in each stage of the wild-type, the homologous gene of Csa1G575100 has been identified to associated to the cell cycle and secondary wall formation (Taylor at al., 2015). On the other hand, some MRDs were also found change expression level in just one stage. For instance, in stage II, the expression level of NTL8 (Csa6G425760) in wild-type was 1.7 fold higher than in mutant, it had been identified that could regulate the development of trichome formation in Arabidopsis (Tian at al., 2017). TDR (Csa4G022350) is a Leucine-rich receptor-like protein kinase that involve in cell division pattern in plant (Yuki and John, 2015), in our transcriptome 
data, its expression level of wild-type was up regulated 1.7 fold in stage III. These results indicate that the genes associated with meristems are widely involved in the difference of spines morphology between wild-type and mutant.

CsTs is a C-type Lectin receptor-like kinase (Guo et al., 2017), its role in the plant is elusive. But studies in mammals show that receptor-like tyrosine protein kinases can identify and receive many growth factors and influence the cell cycle by regulating downstream pathways (Scita et al., 1999). This also partly explains that CsTs may cause phenotypic differences between mutant and wild-type. In our transcriptome data, CsTs had no expression difference between wild-type and mutant, and also had no expression difference in each stage. So the influence of CsTs on downstream networks should be relatively stable. There were 38, 37 and 27 DEGs related to cell cycle in three stages between wild-type and mutant respectively (Table S9), and only 10 were differentially expressed in all three stages (Table S10). Surprisingly, no studies have shown that these 10 genes were involved in the development of trichomes, either in the cucumber or in Arabidopsis, which suggested that the pathways that CsTs acts on may be quite different from the morphology of unicellular trichomes.

\section{The Expression Trend Change of Identified Genes associated to Cucumber Trichomes and Roots Hairs Development}

At present $₫$ just a few genes related to development of cucumber trichomes and roots had been identified (Wang et al., 2015; Sen et al., 2018; Zhao et al., 2015b; Yang et al., 2014; Chen et al., 2016; Hong et al., 2018; Guo et al., 2018), but most of them are transcription factors (table 2). Transcription factors are key nodes of gene regulatory networks, so these genes may provide clues for exploring the network of cucumber spines development. We investigated the change trend of these genes in three stage development of cucumber spines in wild-type and mutant, and found only three genes had different expression trends between wild-type and mutant, they were CsMict, CSMYB6 and CsGA20ox1.

The expression of CsMict did not have significant differences in stage II and stage IV between wild-type and mutant, but in the stage III, wild-type was 1.5 fold higher than mutant. There were 45 genes had the same expression pattern in our transcriptome data (Fig. 9A, B) (Table S11), included some classical genes involved in cell division and differentiation. For example Csa3G730160, up regulated 2.6 fold in wild-type, its homologous gene OFP2 is a transcriptional repressor that can participate in the cell elongation process in Arabidopsis (Zhang et al., 2020). Csa6G106800 was predicted to be involved in cell division and cell circulation, and its homologous gene $A T 2 G 46600$ had been identified regulate the development of trichomes (Marks et al., 2009). In previous study (Zhao et al., 2015b), ten transcription factors had been predicted to be involve in trichomes based on the transcriptome data of CsMict, and we found that four of them also had expression differences in our transcriptome data, and their differences also were stable in the three stages. They were MYB76-like transcription factor Csa3G824850, auxininduced protein Csa2G006270, MYB6-like transcription factor Csa3G168940 and Homeobox-leucine zipper protein Csa7G044240 (Table 3). 
CsMYB6 is a negative regulator to the initiation of cucumber spines, the expression trend of CsMYB6 was consistent between wild-type and mutant, but its expression level in wild-type was significantly higher than in mutant (Fig. 9C, D). Some genes associated with meristem and cell wall modification were found to have the same expression trend with CSMYB6 (Table S11). Such as a bHLH transcription factors Csa5G607470, compared with the wild type, its expression was down-regulated by 1.9, 2.4 and 1.5 fold in each stage of the mutant, the homologous genes of Csa5G607470 can interact with CUC family to affect the development of lateral branch members in Arabidopsis (Tian et al., 2014). CsGA200x 1 is a gibberellin oxidases that had been identified to affect root development in Arabidopsis, the expression of CsGA200x 1 did not have significant different in stage III and stage IV between wild-type and mutant, but in the stage II, wild-type was 2 fold higher than mutant. We found 37 genes had the same expression trend in transcriptome data (Fig. 9E, F) (Table S11), they contained some transcription factors and kinases related to cell differentiation and proliferation. For instance, HLH68 (Csa3G485860), which was down regulated by 2.3 fold in stage II, and its homologous gene (AT4G29100) were involved in regulating fiber differentiation in Arabidopsis (Jürgen et al., 2005). FERONIA (Csa5G640540) approximately down regulates 2 fold in stage II in mutant, and its homologous genes have been shown to affect cell elongation by controlling vacuole expansion (Colette et al., 2010).

\section{The DEGs related to Transcription factors}

Transcription factors are the key nodes of gene regulatory networks, expression level changes of transcription factors will affect the expression levels of many downstream genes. In the different development stage of spines, we identified 161 transcription factors that had expression difference between wild-type and mutant (Table S12). The 161 genes could be subdivided into 26 categories according to their transcription factor family. The most three commonly categories were the ERF-ERF, bHLH and WRKY, they contain 24, 22 and 22 genes respectively (Fig. 10). Among 26 transcription factor families, HD-ZIP (Zhao et al., 2015b; Wang et al., 2015), MYB (Sen at al., 2018) and C2H2 (Yang et al., 2014) have been identified to participated in the development cucumber trichomes, Genes from the same gene family often have similar functions, so we mined the members of three gene families. HD-ZIP transcription factor GLABROUS 2 (Csa7G044240) were up regulated 1.6 and 1.5 fold in mutant in stage II and stage III respectively, trichomes of its homologous gene mutant appear glass-like under a dissecting microscope in Arabidopsis (Schiefelbein 2003; Pesch and Hülskamp 2009). MYB30 (Csa1G009700) were up regulated 2.8, 2.35 and 1.6 fold in mutant in stage II, stage III and stage IV respectively, this gene can regulate elongation of root cell elongation in Arabidopsis (Mabuchi et al., 2018). We also found that other transcription factor families contained some member involved in cell division or multicellular organisms development. For example, TCP4 (Csa4G628330) could regulates cell differentiation (GO: 0030154) and leaf morphogenesis (GO: 0009965), and was up-regulated more than 8 fold in mutant at stage IV, its homologous gene have been reported that can affect trichome cell differentiation (Vadde et al., 2017). Csa6G495620 is one member of GRAS transcription factor, it involved in asymmetric cell division (GO: 0008356) and negative regulation of mitotic cell cycle (GO: 0045930), it was down-regulated 2 fold in mutant at stage II. One NAC family member, Csa6G518170, had higher expression level in wild-type than 
mutant in stage III ( 2 fold) and stage IV ( 15 fold), it involved in multicellular organism development (GO: 0007275) and regulation of secondary cell wall biogenesis (GO: 2000652).

\section{qRT-PCR validation of differentially expressed genes}

In order to validate the accuracy of RNA-seq data, CsMict, CsTu, CsMYB6, Cs2G006270 and Cs3G020060 were selected and subjected to qRT-PCR analysis for three stages. The results showed that data of qRTPCR highly repeated RNA-seq data and thus verified the accuracy of RNA-seq data $(R=0.82 \sim 0.99)$ (Fig. S1).

\section{Discussion}

\section{Development Stages of Fruit Spines}

Trichomes are hairy structure covering the epidermis of cucumber,due to the trichomes on the fruit were harder than other tissues, so this part of trichomes also were called fruit spines. Fruit spines not only have a protective effect to cucumber ovary (Hülskamp et al., 1998; Hülskamp, 2004; Schellmann and Hülskamp, 2005), but also affect the commercial value of cucumber (Chen et al., 2014), so it has been one of the important traits that breeders pay more attention to. Benefit from the fact the cells that make up the fruit spines were larger than other tissue and exist on the surface of the fruit in a low density, we can more easily observe the development of spines morphology in detail by microscope. Therefore, spines provide an excellent model for studying cell differentiation and proliferation (Zhao et al. 2015a). Due to the lack of representative variety materials, previous researches on the development of fruit spines always focused on the presence (Wang et al., 2015), density (Sen et al., 2018) and size (Chen et al., 2014; Zhao et al., 2015b) of fruit spines. There is no precise division of the stages that may be experienced during the development of fruit spines, especially lack of in-depth understanding of the developmental patterns of stalk and base that make up the fruit spines. Fortunately, we have recently obtained a nature mutant that cells of spines have an abnormal division pattern, making it possible to divide important stages in the development of fruit spines.

In this study, we divided the development of fruit spines into stage I to IV (Fig. 2). Stage I mainly involved the specific development of some epidermal cells to initiate the formation of fruit spines. In stage II, stalk of spines begin to develop by a single-cell relay mode, the mark of the end of this stage is the full extension of the fourth single cell of stalk. In stage III, stalk will continues to develop, accompanied by the expansion of the ovary and the rigidity of the fruit spines will increase, the development of stalk will be ended in this stage (7 8cells). Stage IV is mainly the development of the base of spines, the cell population that makes up the base keep dividing and expanding, and eventually form straight and hard fruit spines.

Comparing with the mutant (Fig. 2 and Fig. 3), we found that stage III was the key stage to determine whether the development of fruit spines was abnormal. The wild-type fruit spines will develop a complete stalk at this stage, but the stalk in the mutant stage III is no longer fully extended, the single cells of stalk 
are flat, and even some will divide into multiple cells, which makes the mutant stage IV no longer exist, and the difference between stalk and base is blurred.

\section{CsTs may affect the Development of Fruit Spines together with CsMict}

By combining previous researches (Zhao et al., 2015b) and transcriptome data of this study, we found that due to CsMict is almost not expressed in its mutant, although the mutant of CsMict has trichomes, all cells that make up trichomes undertake an uncomplete development (Fig. S2). But in our transcriptome data, the expression pattern of CsMict only down regulated at the stage III in CsTs mutant, which did not seriously affect the development of the first few single cells and base, just fifth to eighth cells of stalk in CsTs mutant was abnormal (Fig. 3). Therefore, we believe that CsTs may have an impact on CsMict, they are both the impact on the formation of the fruit spines. The expression level of CsMict is related to the development of all cell make up fruit spines, while CsTs mainly affects the development of stalk and has no effect on the apical cell of fruit spines. In addition, we also found that four transcription factors were differentially expressed both in the two transcriptome data, indicating that CsMict and CsTs may have crossover in the pathway of action. In particular, GLABROUS2 (Csa7G044240) has been proved to be closely related to the development of trichomes in plant studies (Schiefelbein 2003; Pesch and Hülskamp 2009). At the same time, there should be more than one regulatory pathway in the process of affecting the development of fruit spines, other genes should also have an impact on CsMict. Previous studies have shown that CsTril has an epistatic effect on CsMict (Wang et al., 2015), and there is no expression level difference of CsTril between wild-type and mutant in our transcriptome data.

\section{The Role of CsTs in the Development of Fruit Spines}

The mutant (Csts) used in this study was initially defined as having a tender fruit spines (Guo et al., 2017). With continuous tracking of the development stage of the fruit spines, we found that the most direct reason for the fruit spines to be more easily to bend is the developmental deformity of the fruit spines in mutant, which makes the combination of base and epidermis become unstable (Fig. 3). Therefore, the role of CsTs, which is localized by map-based cloning, should be the in cell division and differentiation patterns in the development of fruit spines. CsTs is a C-type Lectin receptor-like kinase that located on the cell membrane (Guo et al., 2017). Bioinformatics predicts that it may play an important role in receiving intercellular signals (Guo et al., 2017). Combined with the phenotypic data, the differences of fruit spines between the wild-type and mutant were found in stage III, starting from the fifth single cell of stalk, all single cells that made up stalk would not have a fully elongated, and then the single cells that make up stalk also begin to split into a multi-cell structure similar to base (Fig. 3). So we think that CsTs is responsible for receiving a special signal and maintaining the complete development of the stalk. Once this signal cannot be transmitted normally, the operation of the stalk cell fate will be disordered, leading to the base structure generated in advance. C-type LecRLK is a mysterious gene. Most plants only have one or two this type receptor-like kinase (Vaid et al., 2012; Shumayla et al., 2016; Yang et al., 2016; Liu et al., 2018). Previous studies on model plants have not showed it involved in the development of unicellular trichomes. Studies in mammals have shown that signaling molecules 
mediated by tyrosine protein kinases, including many growth factors, can influence the cell cycle by regulating downstream pathways and even enhance the proliferation of tumor cells (Scita et al., 1999). Therefore, we infer that CsTs performs a similar function in plants. We identified $10 \mathrm{DEGs}$ related to cell cycle between wild-type and mutant in all stages, but there is no studies have shown that these 10 genes were involved in the development of trichomes, either in the cucumber or in Arabidopsis (Table S10). This suggested that CsTs may be involved in a genetic network that we still have insufficient cognition.

\section{Conclusions}

In this study, the development of spines was divided into four stages by phenotypic changes during developmental process. Based on analysis of transcriptome data, a number of DEGs related to cell cycle and meristem were identified. By correlating the expression patterns of several transcription factors that have been reported to be involved in the development of spines, we identified some genes that may be involved in the formation of cucumber fruit spines. We also found 26 transcription factor families may be involved in the development of fruit spines. The results provided a step stone for further analysis of the molecular mechanism in the developmental process of multicellular trichomes.

\section{Materials And Methods}

\section{Plant materials}

The North China type 'NC072' (wild-type) and its inbred spines mutant 'NC073' were provided by the Tianjin Derit Seeds Company Ltd (Tianjin, China), NC072 and NC073 share almost the same genetic background. Cucumber plants were grown with appropriate management in a greenhouse (Shanghai, China) in the autumn of 2019, under natural photoperiodic conditions. Fruit spines from wild-type and mutant cucumber fruit that were $0.2,0.35,0.6 \mathrm{~cm}$ in length were isolated under a dissecting microscope, and spines from at least fifteen fruits from different plants were pooled as one biological sample. Three biological replications were performed for each set of experiments.

\section{Morphology Observation of fruits and spines}

The SEM analyses of samples were performed according to Wang et al (2016). Digital photos of cucumber fruit in different lengths were taken by Canon EOS Digital camera. Photos of Microscope were taken by LECIA DM 2500.

\section{Total RNA extraction, library construction and Illumina sequencing}

Total RNA was extracted using an RNeasy Plant Mini Kit (Cwbio, Beijing, China). RNA quantity and quality were analysed using the Agilent Bioanalyzer 2100 system (US). The high-quality RNA samples were sequenced by Biomarker Bioinformatics Technology Co., Ltd (Beijing, China), and cDNA library construction and Illumina HiSeq ${ }^{\text {TM }} 2500$ were performed as described previously (Wei et al., 2016). 


\section{Sequence assembly of RNA-Seq}

The raw reads were first filtered to identify clean reads by removing the reads with only adaptor sequences and unknown nucleotides $>5 \%$, as well as low quality reads (percentage of low-quality bases with a quality value $\leq 5$ in more than $50 \%$ of a read). Clean reads were mapped to the cucumber genome sequence (Cucumber 9930 genome v2) (ftp://www.icugi.org/pub/cucurbit/genome/cucumber/ Chinese_long/v2/) using the HISAT2 software.

\section{Differential Expression Genes (DEGs) Analysis}

FPKM (Fragments Per Kilobase of transcript per Million fragments mapped) values (Florea et al., 2013) were used to analyze gene expression by the StringTie software (Pertea et al., 2015). The $P$ value $<0.05$ and fold change $\geq 1.5$ were chosen as the threshold of DEGs.

\section{Functional Analysis of DEGs}

The functional of DEGs were identified using BLASTX program (Altschul et al., 2007) against several classic biological databases, they are NCBI non-redundant protein database (NR http://www.ncbi.nlm.nih.gov), Gene Ontology (GO, http: //www.geneontology. org/), Kyoto Encyclopedia of Genes and Genomes (KEGG, http://www.genome.jp/kegg/), Swiss-Prot (http://web.expasy.org/docs/relnotes/relstat.html), Clusters of Orthologous Groups (COG, http://www.ncbi.nlm.nih. gov) and the Pfam (http://pfam.xfam.org/), using a cut-off Evalue of $10^{-5}$. Then we assigned DEGs to KEGG pathways by KOBAS 2.0 software (Xie et al., 2011). TopGO software (Alexa and Rahnenfuhrer, 2010) was used to plot GO functional classification for the DEGs with GO term hits to view the distribution of gene functions of the species at the macro level.

\section{Real-time qPCR analysis}

In order to estimate the accuracy of RNA-Seq, we performed Quantitative RT-PCR (qRT-PCR) using the same RNA samples which were used for RNA library construction. The first strand cDNA was prepared according to the PrimeScript RT reagent Kit with gDNA Eraser (Cwbio, Beijing, China) protocol. qRT-PCR was conducted using FastStart Essential DNA Green Master (Roche, Mannheim, Germany). CsActin3 (Csa6G484600.1) was used as an internal control. qRT-PCR was performed in a total volume of $20 \mu \mathrm{L}$, containing $2 \mu \mathrm{L}$ of cDNA, $10 \mu \mathrm{L}$ SYBR mix, $2 \mu \mathrm{L}$ gene-specific primers $(10 \mu \mathrm{M})$ and $6 \mu \mathrm{ddH}_{2} \mathrm{O}$, using the CFX Connection Real-Time System (Bio-Rad, California, USA) with 40 cycles of $5 \mathrm{~s}$ at $95^{\circ} \mathrm{C}, 30 \mathrm{~s}$ at $60^{\circ} \mathrm{C}$. Three biological replicates were analyzed for each sample. Geneious software (version 2019.0.3) was used to design primers according to the cDNA sequences (Table S1). The data from real-time PCR amplification was analyzed using $2^{-} \triangle \triangle C T$ method at first (Livak and Schmittgen, 2000).

\section{Abbreviations}


Wt: wild-type, Mu: mutant, DEGs: Differential Expression Genes, FPKM: Fragments Per Kilobase of transcript per Million fragments mapped, MRDs: DEGs involved in meristem regulation, GL1: GLABRA1, MYB: v-myb avian myeloblastosis viral oncogene homolog, TTG1: TRANSPARENT TESTA GLABRA1, bHLH: basic helix-loop-helix, GL3: GLABRA3, EGL3: ENHANCER OF GLABRA3, TRY: TRIPTYCHON, CPC: CAPRICE, ETC: ENHANCER OF TRY AND CPC, SEM: Scanning Electron Microscope, Tril: trichome-less, Tu: tuberculate fruit, Ts: tender spine, LecRLK: Lectin receptor-like kinase, Mict: Micro-trichome, GO: Gene Ontology, KEGG: Kyoto Encyclopedia of Genes and Genomes, COG: Clusters of Orthologous Groups, NR: non-redundant.

\section{Declarations}

\section{Ethics approval and consent to participate}

Not applicable, as this study did not involve human or animal subjects, and the seeds of cucumber were stored in School of Agriculture and Biology, Shanghai Jiao Tong University.

The experiments comply with the current laws of the country in which we were performed.

\section{Availability of data and materials}

All data generated or analysed during this study are included in this published article [and its supplementary information files].

\section{Consent for publication}

Not applicable.

\section{Competing interests}

The authors declare that they have no conflict of interest.

\section{Funding}

This study was supported by the Project of Science and Technology Commission of Shanghai Municipality (No: 18391900300), it supported us in RNA sequencing.

This study was supported by the Agri-X Prolu0002ject of Shanghai Jiao Tong University (Agri-X2017011), it supported us in experiment of data analysis and essay writing.

This research was supported by the National Natural Science Foundation of China (No. 31672148), this foundation supported us in qRT-PCR

\section{Authors' contributions}


DL contributed to bioinformatics analysis and writing of the manuscript. LRX and YY helped with the RNA extraction and qRT-PCR. HLH, JXS and KYZ helped with growing plants. JAP contributed to plant materials. RC, GW and JSP provided critical insights and revised the manuscript. All authors read and approved the final manuscript.

\section{Acknowledgements}

We thank Qi Zhang (Agriculture Commission, Jinshan District, China) for revising the manuscript.

\section{References}

Alexa A, Rahnenfuhrer J. topGO: enrichment analysis for gene ontology. R package version 2.8, 2010.

Altschul S F, Madden T L, Schffer A A, et al. Gapped BLAST and PSI-BLAST: a new generation of protein database search programs.[J]. Nuclc acids research, 1997, 25(17):3389.

Chen C, Liu M, Jiang L, et al. Transcriptome profiling reveals roles of meristem regulators and polarity genes during fruit trichome development in cucumber (Cucumis sativus L.)[J]. Journal of Experimental Botany, 2014, 65(17):4943-4958.

Chen C, Yin S, Liu X, et al. CsTTG1 Encodes a WD-repeat Protein That Regulates Fruit Wart Formation in Cucumis sativus through Interaction with the Homeodomain-leucine Zipper I Protein Mict[J]. Plant Physiology, 2016: pp. 00112.2016.

Chen X, Xizeng M, Jiaju $\mathrm{H}$, et al. KOBAS 2.0: a web server for annotation and identification of enriched pathways and diseases[J]. Nucleic Acids Research(suppl_2):suppl_2.

Colette A. ten Hove, Viola Willemsen, et al. Encodes a Nuclear Factor Regulating Asymmetry of Stem Cell Divisions in the Arabidopsis Root[J]. Current Biology, 2010, 20, 452-457.

Esch J J, Chen M A, Hillestad M, et al. Comparison of TRY and the closely related At1g01380 gene in controlling Arabidopsis trichome patterning[J]. Plant Journal, 2004, 40(6):860-869.

Florea, Liliana, Song, Li, Salzberg, Steven L. Thousands of exon skipping events differentiate among splicing patterns in sixteen human tissues[J]. f1000res, 2013, 2:188.

Galway M E, Masucci J D, Lloyd A M, et al. The TTG Gene Is Required to Specify Epidermal Cell Fate and Cell Patterning in the Arabidopsis Root[J]. Developmental Biology, 1994, 166(2):0-754.

Hong Sun, Baoya Pang, Jun Yan, et al. Comprehensive Analysis of Cucumber Gibberellin Oxidase Family Genes and Functional Characterization of CsGA20ox1 in Root Development in Arabidopsis [J]. Journal of Molecular Sciences, 2018, 19, 3135. 
Guo C, Yang X, Wang Y, et al. Identification and mapping of ts (tender spines), a gene involved in soft spine development in Cucumis sativus[J]. Theoretical and Applied Genetics, 2017.

Hülskamp M, Schnittger A, Folkers U. Pattern formation and cell differentiation: trichomes in Arabidopsis as a genetic model system[J]. International Review of Cytology, 1998, 186(4):147-150.

Hülskamp, Martin. Plant trichomes: a model for cell differentiation[J]. Nat Rev Mol Cell Biol, 2004, 5(6):471-480.

Johnson, C. S. TRANSPARENT TESTA GLABRA2, a Trichome and Seed Coat Development Gene of Arabidopsis, Encodes a WRKY Transcription Factor[J]. THE PLANT CELL ONLINE, 2002, 14(6):1359-1375.

Jürgen Ehlting, Mattheus N , Aeschliman D S , et al. Global transcript profiling of primary stems from Arabidopsis thaliana identifies candidate genes for missing links in lignin biosynthesis and transcriptional regulators of fiber differentiation[J]. The Plant Journal, 2005, 42.

Kirik V, Simon M, Huelskamp M, et al. The ENHANCER OF TRY AND CPC1 gene acts redundantly with TRIPTYCHON and CAPRICE in trichome and root hair cell patterning in Arabidopsis[J]. developmental biology, 2004, 268(2):0-513.

Kirik V, Simon M, Wester K, et al. ENHANCER of TRYandCPC 2(ETC2) reveals redundancy in the regionspecific control of trichome development of Arabidopsis[J]. Plant Molecular Biology, 2004, 55(3):389-398.

Li Q, Cao C, Zhang C, Zheng S, et al. The identification of Cucumis sativus Glabrous 1 (CsGL1) required for the formation of trichomes uncovers a novel function for the homeodomain-leucine zipper I gene[J]. Journal of Experimental Botany, 2015, 66: 2515-2526.

Livak K, Schmittgen T. Analysis of Relative Gene Expression Data Using Real-Time Quantitative PCR and the $2^{-\triangle \Delta C t}$ Method[J]. Methods, 2000, 25 (4).

Mabuchi K, Maki H, Itaya T, et al. MYB30 links ROS signaling, root cell elongation, and plant immune responses[J]. Proc Natl Acad Sci U S A, 2018, 115(20): E4710.

Marks M D, Wenger J P, Gilding E, et al. Transcriptome Analysis of Arabidopsis Wild-Type and gl3-sst sim Trichomes Identifies Four Additional Genes Required for Trichome Development[J]. Molecular Plant, 2009, 2(4):p.803-822.

M. Taylor, L. Lin, M. de Lucas et al. An Arabidopsis gene regulatory network for secondary cell wall synthesis[J]. Nature, 2015, 517: 571-575.

Nikovics K, Blein T, Peaucelle A, et al. The Balance between the MIR164A and CUC2 Genes Controls Leaf Margin Serration in Arabidopsis[J]. Plant Cell, 2006, 18(11):2929-2945. 
Oppenheimer D G, Herman P L, Sivakumaran S, et al. A myb gene required for leaf trichome differentiation in Arabidopsis is expressed in stipules[J]. cell, 1991, 67(3):483-493.

Pan Y, Bo K, Cheng Z, Weng Y. The loss-of-function GLABROUS 3 mutation in cucumber is due to LTRretrotransposon insertion in a class IV HD-ZIP transcription factor gene CsGL3 that is epistatic over CsGL1[J]. Bmc Plant Biology, 2015, 15(1):302.

Patrick Laufs, Alexis Peaucelle, Halima Morin, et al. MicroRNA regulation of the CUC genes is required for boundary size control in Arabidopsis meristems. [J]. Development, 2004, 131, 4311-4322.

Payne C T, Zhang F, Lloyd A M. GL3 encodes a bHLH protein that regulates trichome development in Arabidopsis through interaction with GL1 and TTG1[J]. Genetics, 2000, 156(3):1349-1362.

Pertea M, Pertea G M, Antonescu C M, et al. StringTie enables improved reconstruction of a transcriptome from RNA-seq reads[J]. Nature Biotechnology, 2015, 33(3):290-295.

Pesch M, Hülskamp M. One, two, three...models for trichome patterning in Arabidopsis?[J]. Current Opinion in Plant Biology, 2009, 12(5):587-592.

Pesch M, Hülskamp M. Role of TRIPTYCHON in trichome patterning in Arabidopsis[J]. BMC Plant Biology $2011,11,1471-2229$.

Pesch M, Hulskamp M. Creating a two-dimensional pattern de novo during Arabidopsis trichome and root hair initiation [Review][J]. Current Opinion in Genetics \& Development, 2004, 14(4):p.422-427.

Scita, Giorgio, Nordstrom, et al. EPS8 and E3B1 transduce signals from Ras to Rac.[J]. Nature, 1999, 401 (6750): 290-293.

Sen Y, Yanling C, Xingwang L, et al. A CsMYB6-CsTRY module regulates fruit trichome initiation in cucumber[J]. Journal of Experimental Botany, 2018, 69(8): 1887-1902.

Schellmann S, Schnittger A, Kirik V, et al. TRIPTYCHON and CAPRICE mediate lateral inhibition during trichome and root hair patterning in Arabidopsis[J]. Embo Journal, 2002, 21(19):5036-5046.

Schiefelbein $\mathrm{J}$. Cell-fate specification in the epidermis: a common patterning mechanism in the root and shoot[J]. 2003, 6(1):74-78.

Simon M, Lee M M, Lin Y, et al. Distinct and overlapping roles of single-repeat MYB genes in root epidermal patterning[J]. Developmental Biology, 2007, 311(2):0-578.

Shumayla, Shailesh S , Pandey A K, et al. Molecular Characterization and Global Expression Analysis of Lectin Receptor Kinases in Bread Wheat (Triticum aestivum)[J]. PLOS ONE, 2016, 11(4):e0153925.

Szymanski D B, Lloyd A M, Marks M D. Progress in the molecular genetic analysis of trichome initiation and morphogenesis in Arabidopsis[J]. Trends in Plant Science, 2000, 5(5):214-219. 
Szymanski D B. Control of GL2 expression in Arabidopsis leaves and trichomes[J]. Development, 1998, 125.

Tian C, Zhang X, He J, et al. An organ boundary-enriched gene regulatory network uncovers regulatory hierarchies underlying axillary meristem initiation[J]. Molecular Systems Biology, 2014, 10(10):755-755.

Tian $\mathrm{H}$, Wang X, Guo H, et al. NTL8 regulates trichome formation in Arabidopsis by directly activating R3 MYB genes TRY and TCL1[J]. Plant Physiology, 2017:pp.00510.2017.

Vadde B V L, Challa K R, Nath U. The TCP4 transcription factor regulates trichome cell differentiation by directly activating GLABROUS INFLORESCENCE STEMS in Arabidopsis thaliana[J]. Plant Journal, 2017, 93(2).

Vaid N, Pandey P K, Tuteja N. Genome- wide analysis of lectin receptor-like kinase family from Arabidopsis and rice[J]. Plant Molecular Biology, 2012, 80(4-5): 365-388.

Wada, T. Role of a positive regulator of root hair development, CAPRICE, in Arabidopsis root epidermal cell differentiation[J]. Development, 2002, 129(23):5409-5419.

Walker A R, Davison P A, Bolognesi-Winfield A C, et al. The TRANSPARENT TESTA GLABRA1 Locus, Which Regulates Trichome Differentiation and Anthocyanin Biosynthesis in Arabidopsis, Encodes a WD40 Repeat Protein[J]. The Plant Cell, 1999, 11(7):1337-1349.

Wang Y L, Nie J T, Chen H M, et al. Identification and mapping of Tril, a homeodomain-leucine zipper gene involved in multicellular trichome initiation in Cucumis sativus[J]. Theoretical and Applied Genetics, 2015, 129(2):305.

Werker E . Trichome diversity and development[J]. Advances in Botanical Research, 2000, 31:1-35.

Wester K, Digiuni S, Geier F, et al. Functional diversity of R3 single-repeat genes in trichome development[J]. Development, 2009, 136(9):1487-1496.

Xiaowei Zhang, Jiali Wua., Qin Yu, et al. AtOFPs regulate cell elongation by modulating microtubule orientation via direct interaction with TONNEAU2[J]. Plant Science, 2020, 292, 110405.

Yang X, Zhang W, He H, et al. Tuberculate fruit gene Tu encodes a $\mathrm{C} 2 \mathrm{H} 2$ zinc finger protein that is required for the warty fruit phenotype in cucumber ( Cucumis sativus L.) [J]. Plant Journal, 2014, 78(6).

Yang Y, Labbé, Jessy, Muchero W, et al. Genome-wide analysis of lectin receptor-like kinases in Populus[J]. BMC Genomics, 2016, 17(1):699.

Yuki Hirakawa and John L. Bowman. A Role of TDIF Peptide Signaling in Vascular Cell Differentiation is Conserved Among Euphyllophytes[J]. Frontiers in Plant Science, 2015, 6. 
Zhang, F. A network of redundant bHLH proteins functions in all TTG1-dependent pathways of Arabidopsis[J]. Development, 2003, 130(20):4859-4869.

Zhao JL, Wang YL, Yao DQ, et al. Transcriptome profiling of trichome-lessreveals genes associated with multicellular trichome development in Cucumis sativus[J]. Molecular Genetics \& Genomics, 2015a, 290(5):2007-2018.

Zhao J L, Pan J S, Guan Y, et al. Micro-trichome as a class I homeodomain-leucine zipper gene regulates multicellular trichome development in Cucumis sativus[J]. Journal of Integrative Plant Biology, 2015b, 057(011):925-935.

\section{Tables}

Due to technical limitations, Tables 1-3 are only available as a download in the supplemental files section.

\section{Table titles}

Table 1 The summary of RNA-Seq data

(a-c) Three biological replications

Table 2 Identified genes related to development to trichomes and root hair Abbreviations: FC. Fold Change.

Table 3 The predicted genes that were repeated in both studies

Abbreviations: FC. Fold Change.

\section{Supplement Materials}

Additional file 1: Table S1. Primers for qRT-PCR in this study. (XLSX 303 kb)

Additional file 2: Table S2. The DEGs in this study (XLSX 2,886 kb)

Additional file 3: Table S3. KEGG analysis of all DEGs in wild type (XLSX $23 \mathbf{~ k b )}$

Additional file 4: Table S4. KEGG analysis of all DEGs in mutant (XLSX 21 kb)

Additional file 5: Table S5. KEGG analysis of all DEGs between wild type and mutant (XLSX 22 kb)

Additional file 6: Table S6. MRDs between wild-type and mutant in the stage II (XLSX 30 kb) 
Abbreviations: MRDs: genes involved in meristem regulation from DEGs, FC. Fold Change, FPKM: Fragments Per Kilobase of transcript per Million fragments mapped.

Additional file 7: Table S7. MRDs between wild-type and mutant in the stage III (XLSX 30 kb) Abbreviations: MRDs: genes involved in meristem regulation from DEGs, FC. Fold Change, FPKM: Fragments Per Kilobase of transcript per Million fragments mapped.

Additional file 8: Table S8. MRDs between wild-type and mutant in the stage IV (XLSX $30 \mathrm{~kb}$ ) Abbreviations: MRDs: genes involved in meristem regulation from DEGs, FC. Fold Change, FPKM: Fragments Per Kilobase of transcript per Million fragments mapped.

Additional file9: Table S9. DEGs related to cell cycle between wild-type and mutant (XLSX 38 kb)

Additional file10: Table S10. DEGs related to cell cycle between wild-type and mutant in all stages (XLSX $14 \mathrm{~kb})$

Additional file11: Table S11. The Expression trend change of identified genes associated to cucumber trichomes and roots development (XLSX 82 kb)

Additional file12: Table S12. The transcription factors from DEGs between wild-type and mutant (XLSX $50 \mathrm{~kb}$ )

Additional file13: Figure S1 qRT-PCR Analysis of selected genes (PDF 586 kb)

The blue line represents FPKM (Fregments Per Kilobase per Million) and the red line represents the expression levels analyzed by qRT-PCR; pearson correlation coefficients $\mathrm{R}$ range between 0.82 and 0.99 and averaged 0.979 .

(A, C, E, G, I): Wild-type; (B, D, F, H, J): Mutant.

Additional file14: Figure S2. Trichomes on mutant of CsMict (Chen et al., 2014) (PDF 2,935 kb)

\section{Figures}



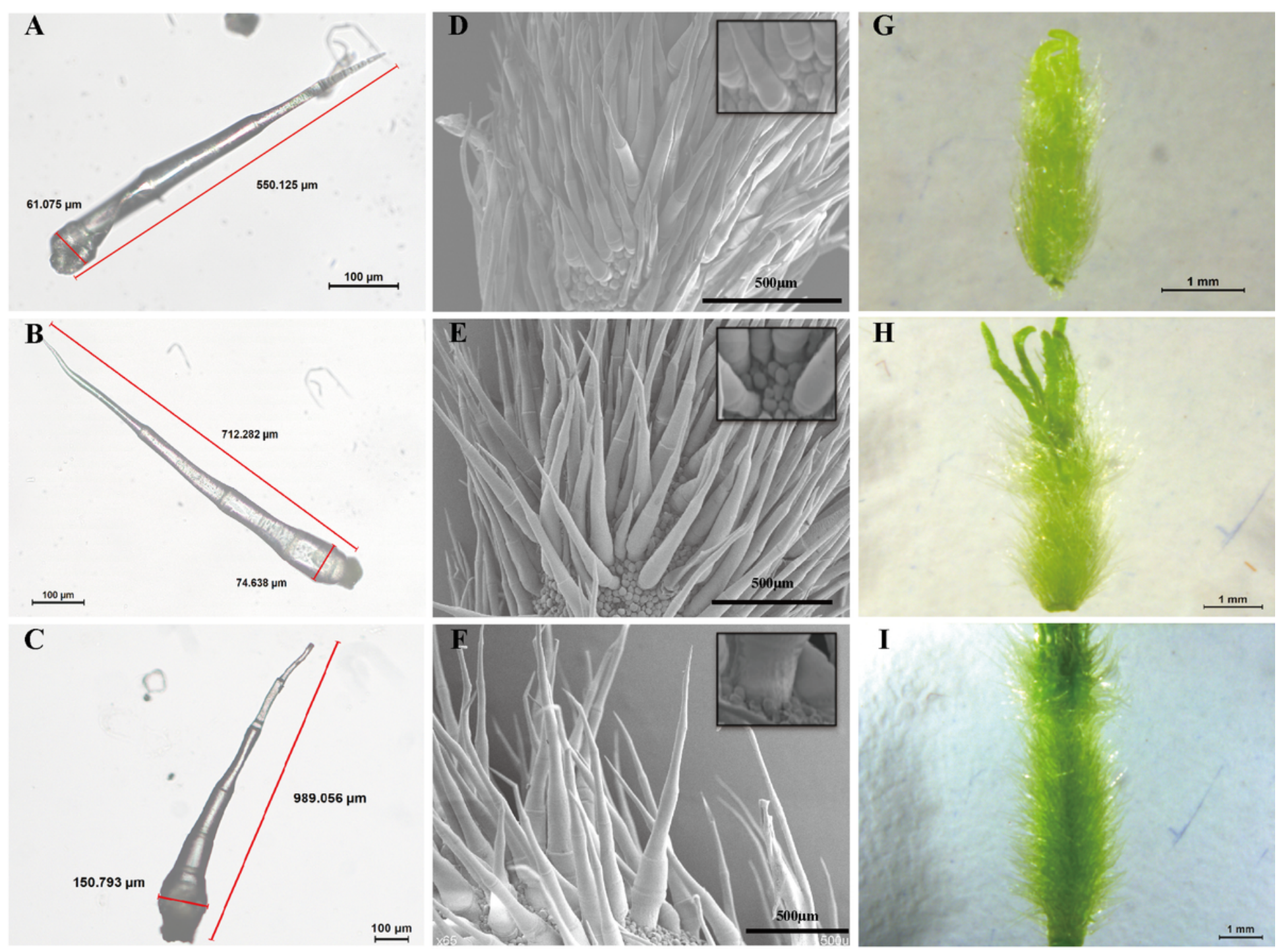

\section{Figure 1}

Key developmental stages of wild type spines. (A-C) Microscope image; (D-F) SEM images; (G-I) Digital camera images Microscope; (A, D, G) Stage II of spines development; $(B, E, H)$ Stage III of spines development; (C, F, I) Stage IV of spines development. 

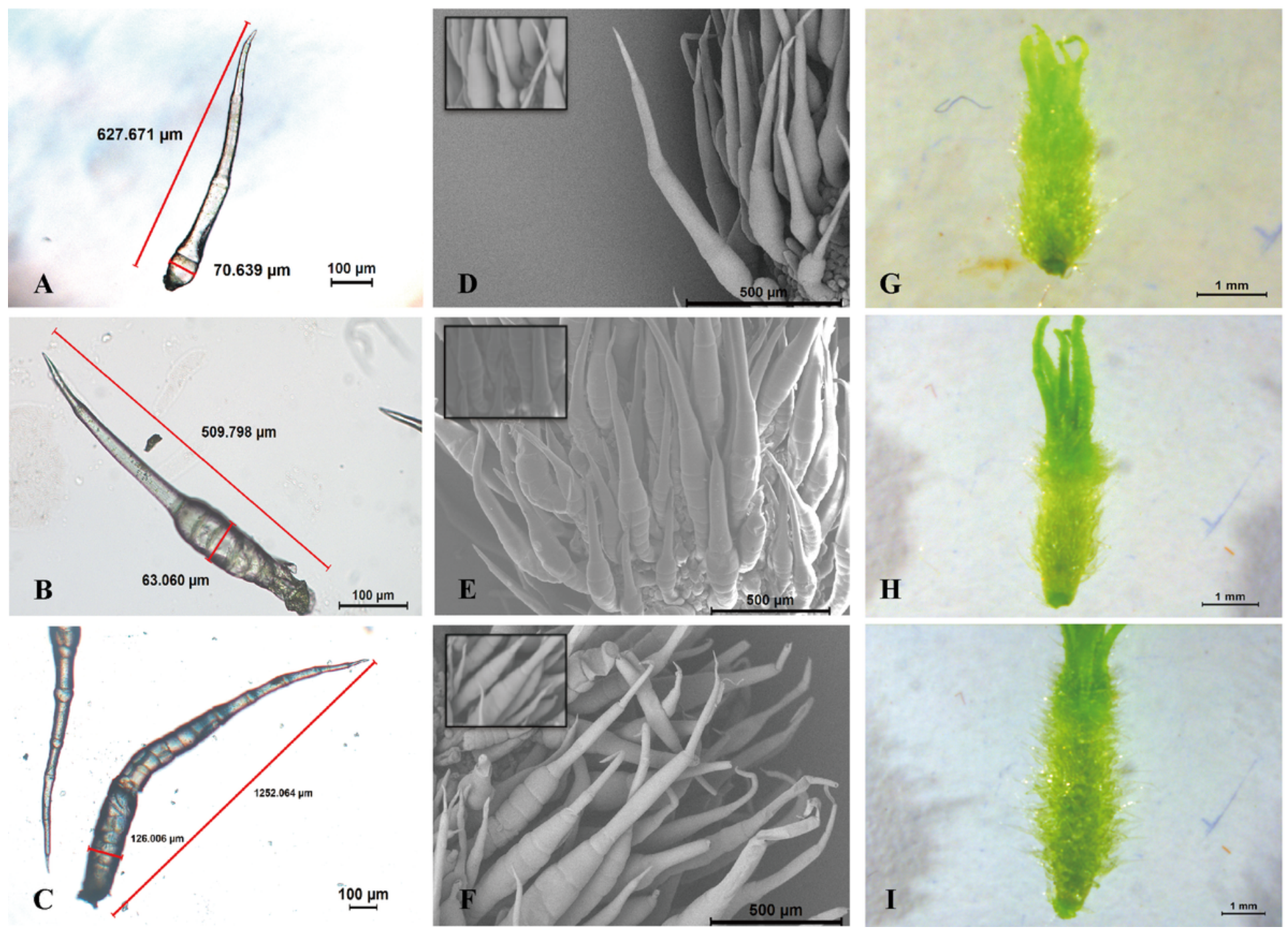

Figure 2

Key developmental stages of mutant spines. (A-C) Microscope image; (D-F) SEM images; (G-I) Digital camera images Microscope; (A, D, G) Stage II of spines development; $(B, E, H)$ Stage III of spines development; (C, F, I) Stage IV of spines development. 

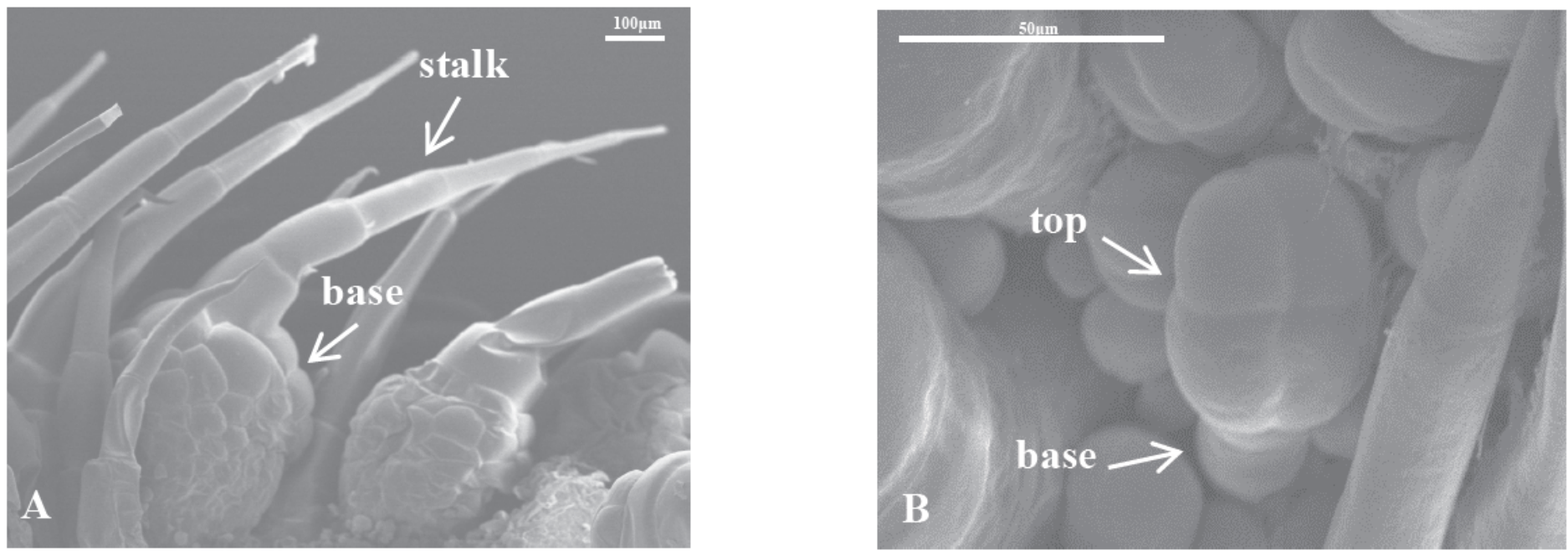

\section{Figure 3}

Morphological characterization of two type trichomes of cucumber. (A) Type I trichomes, (B) Type II trichomes.
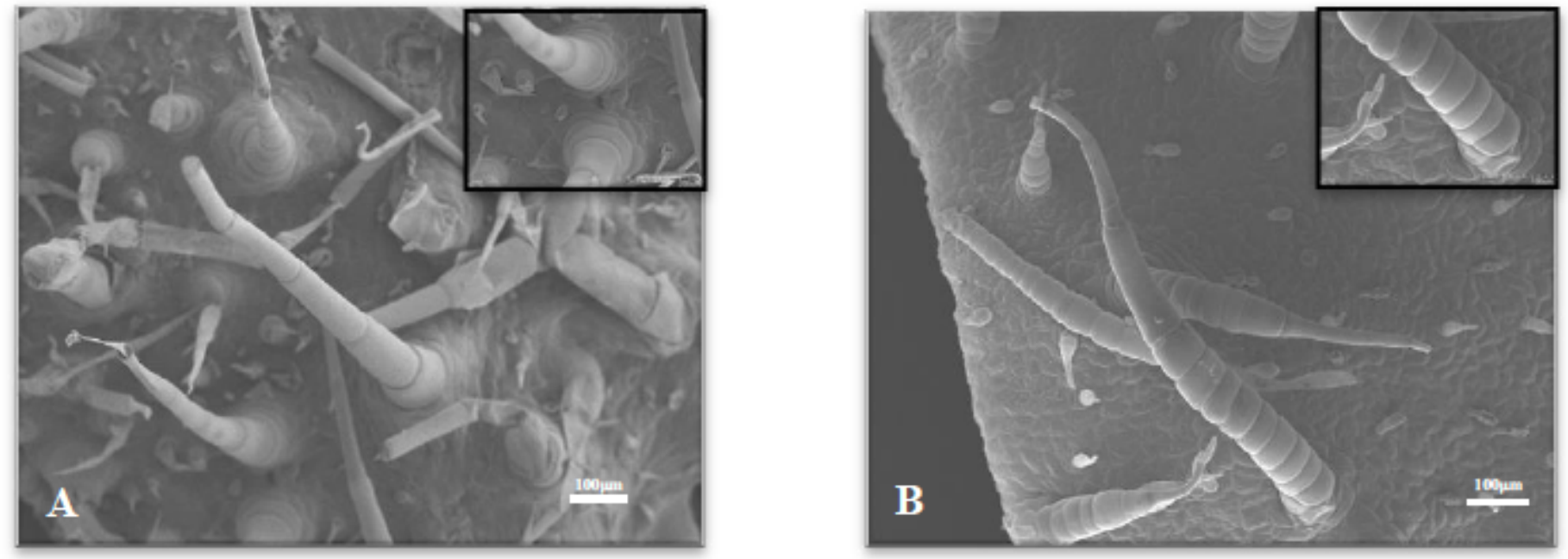

\section{Figure 4}

Morphological characterization of trichomes of receptacles. (A) Wild type; (B) Mutant. 

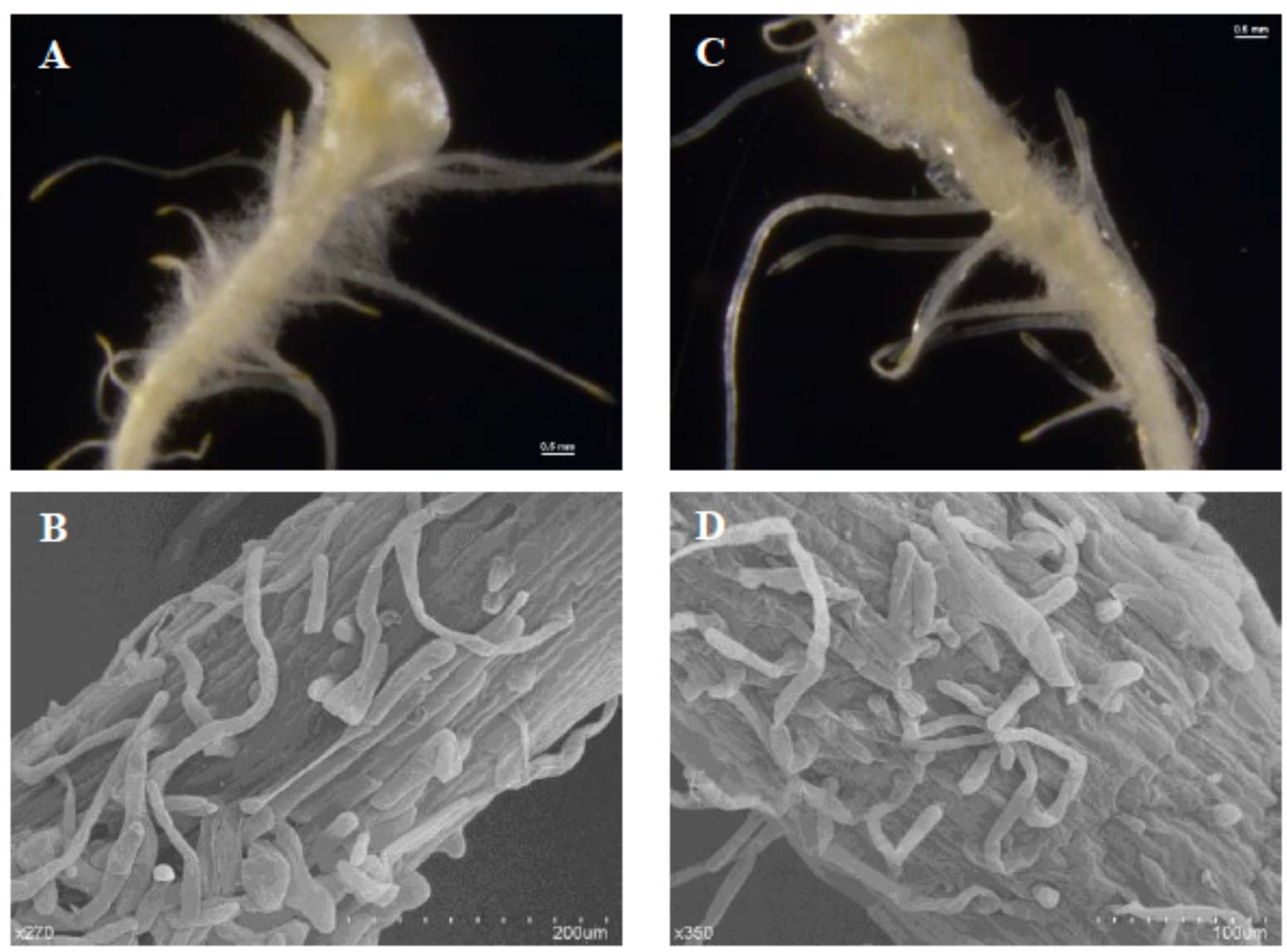

Figure 5

Morphological characterization of root hair. (A-C) Microscope image; (B-D) SEM images; (A, B): Wild type; (C, D): Mutant. 
A Correlation between different Sample of Wild type

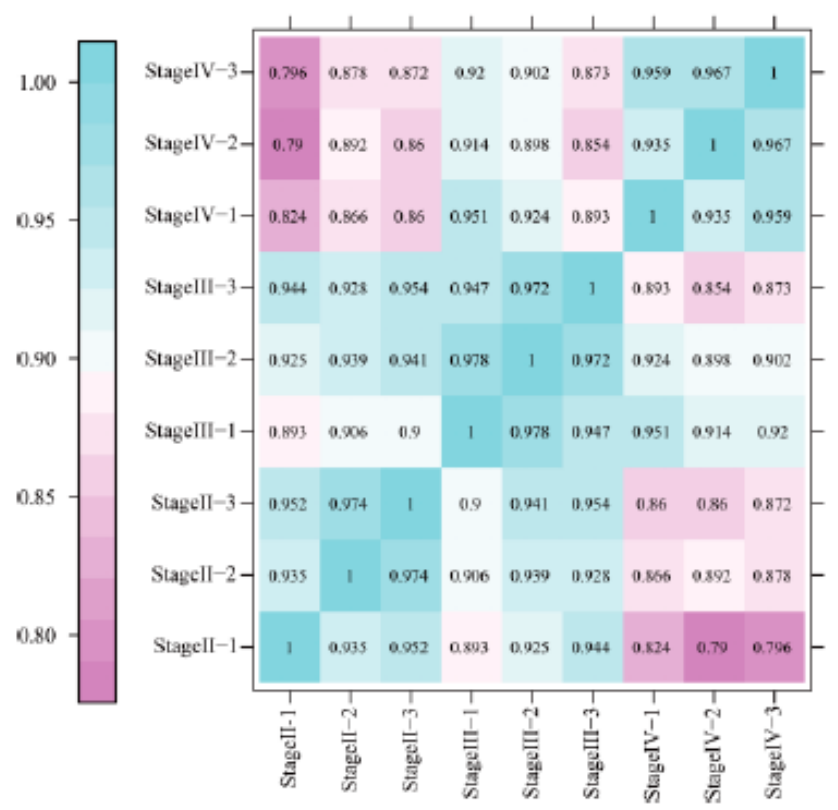

B

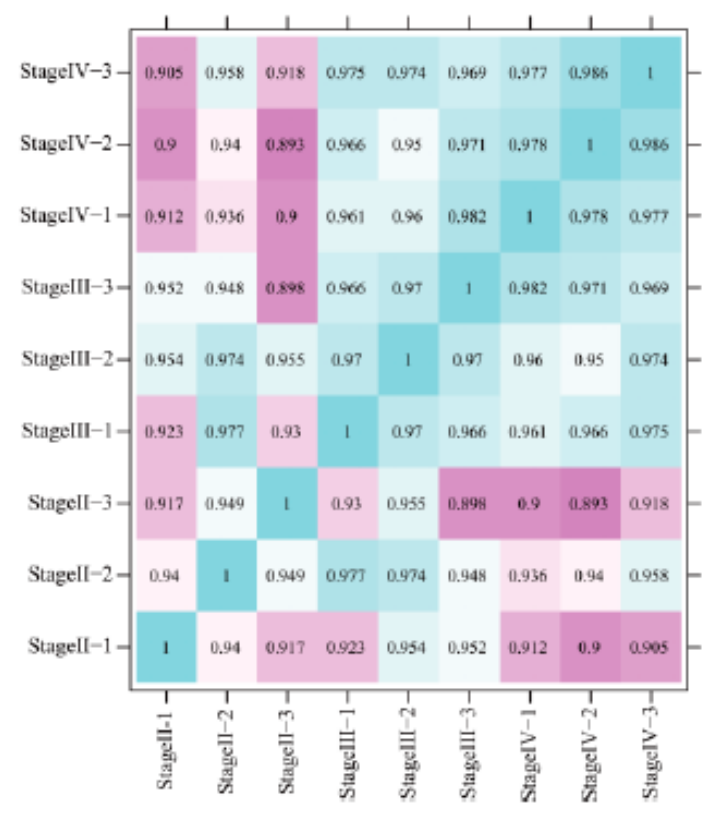

Figure 6

Correlation coefficients for every two samples. (A) Wild-type; (B) Mutant. 

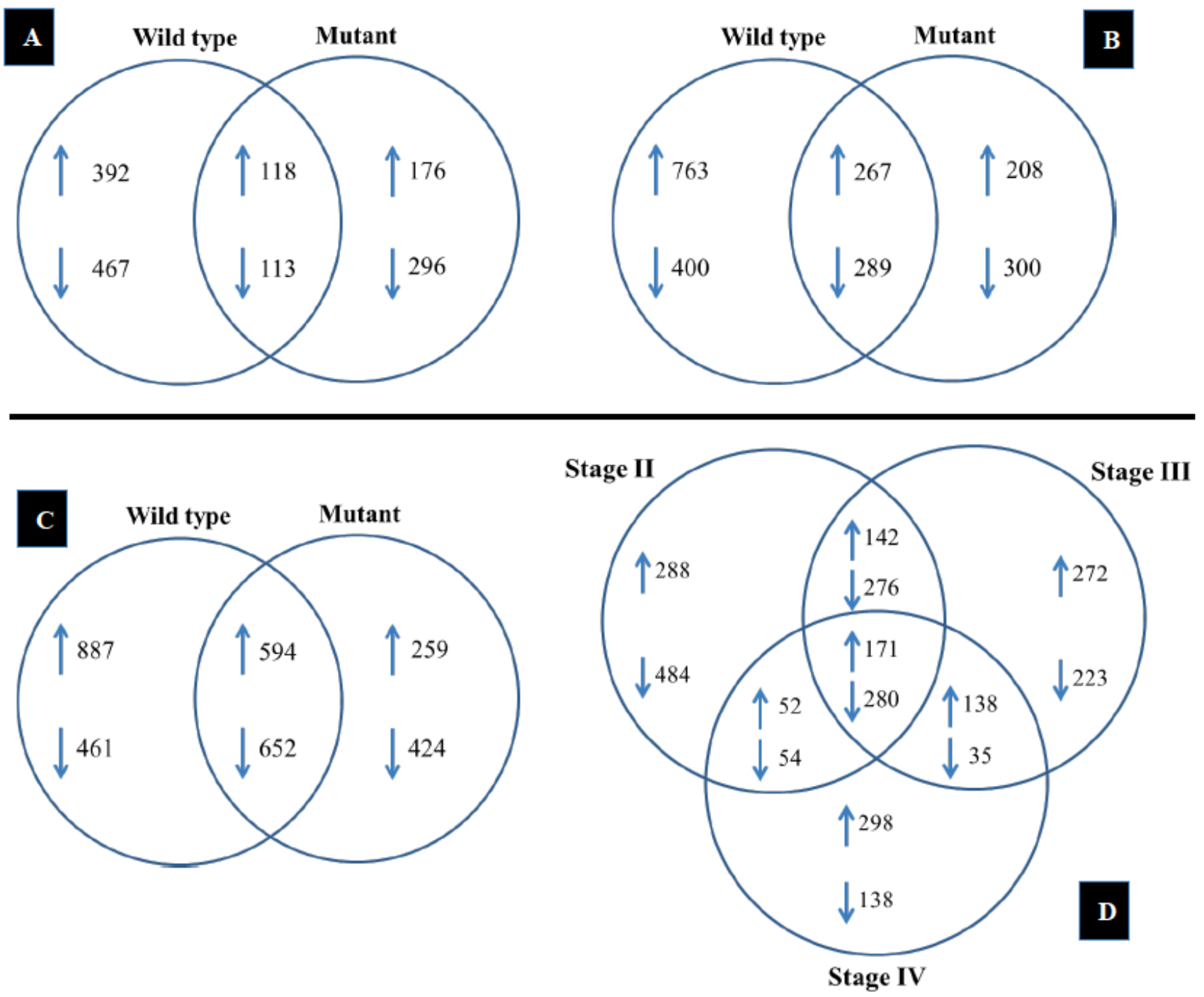

Figure 7

Summary of DEGs. (A) Stage III vs Stage II, (B) Stage IV vs Stage II, (C) Stage IV vs Stage III, (D) DEGs in different Stage between Wild type and mutant. 

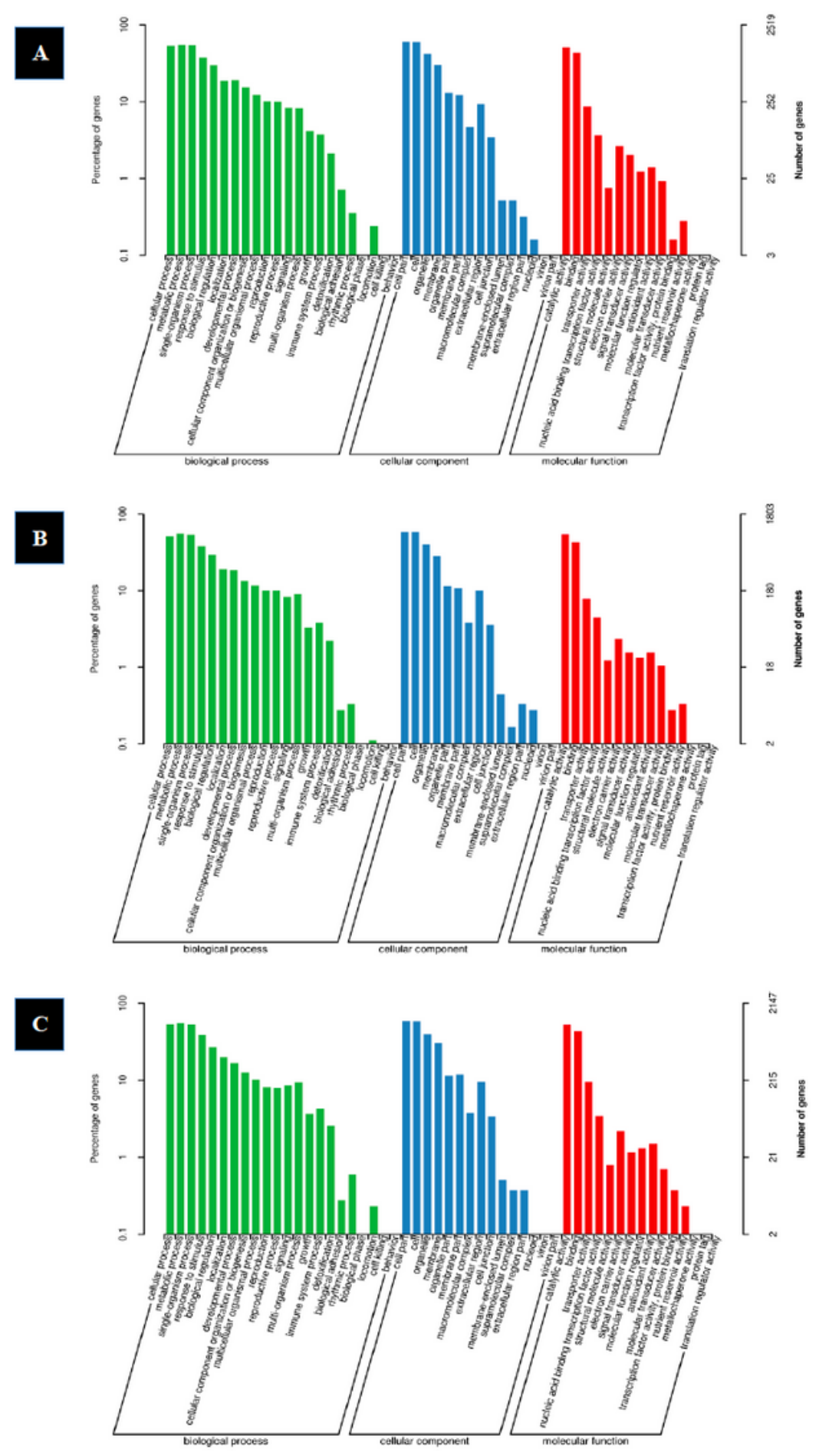

\section{Figure 8}

Histogram presentation of Gene Ontology (GO) classifications. Left y-axis indicates the percentage of DEGs in subcategories of each main category. Right y-axis indicates the number of DEGs in each subcategory. (A) all DEGs of Wild-type, (B) all DEGs of Mutant, (C) all DEGs between Wild-type and Mutant. 


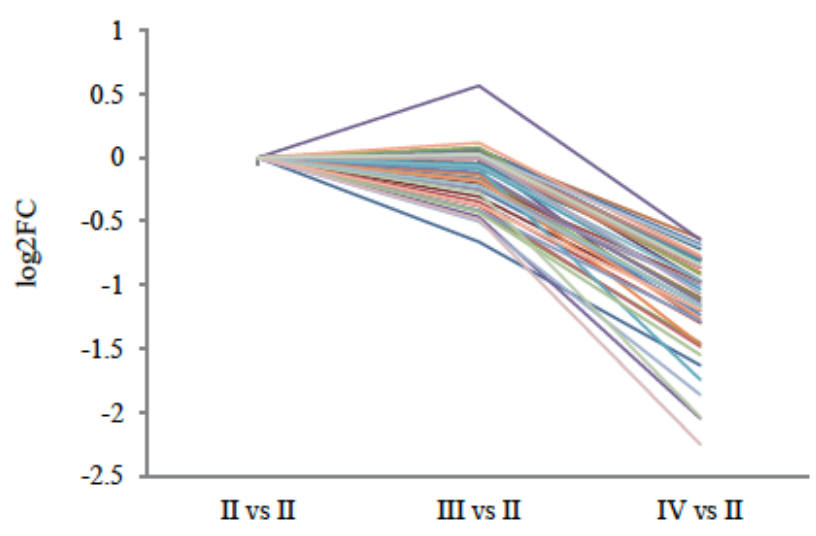

A
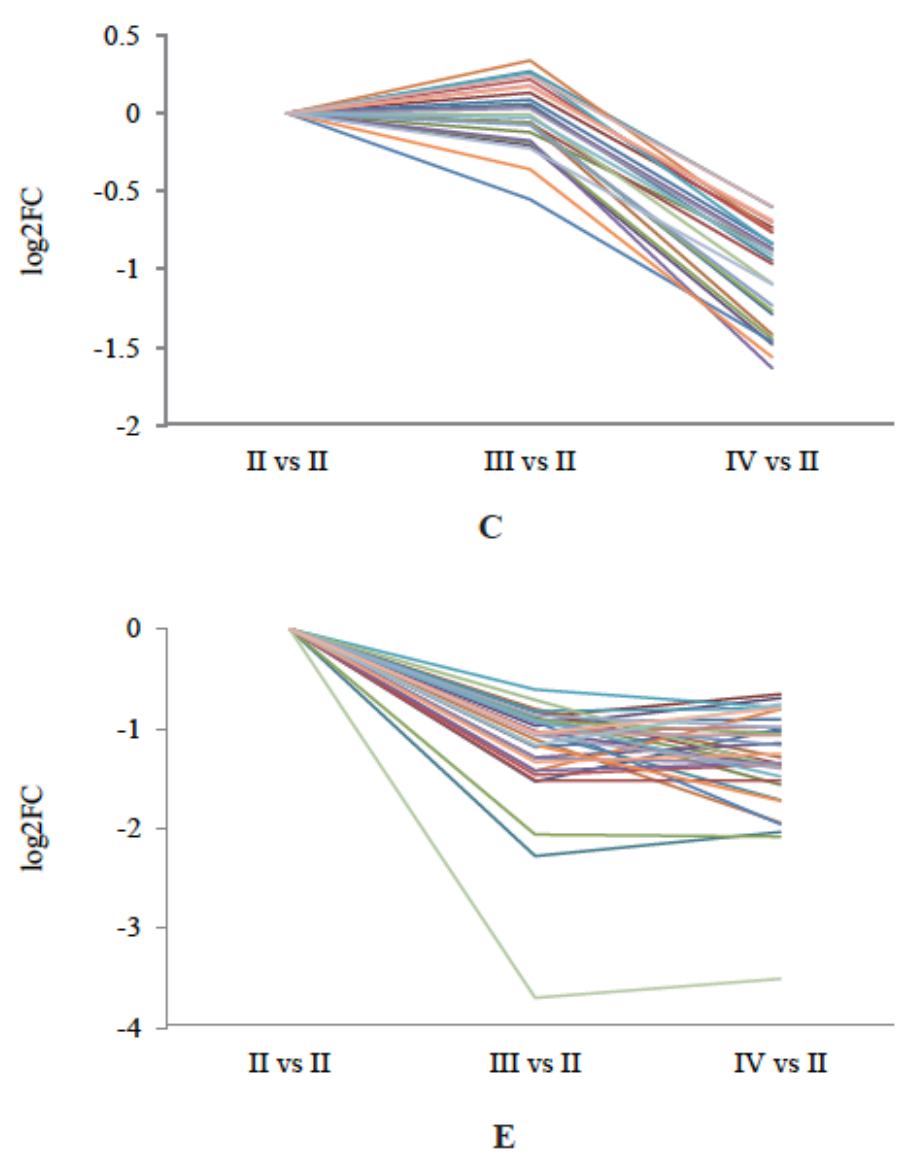
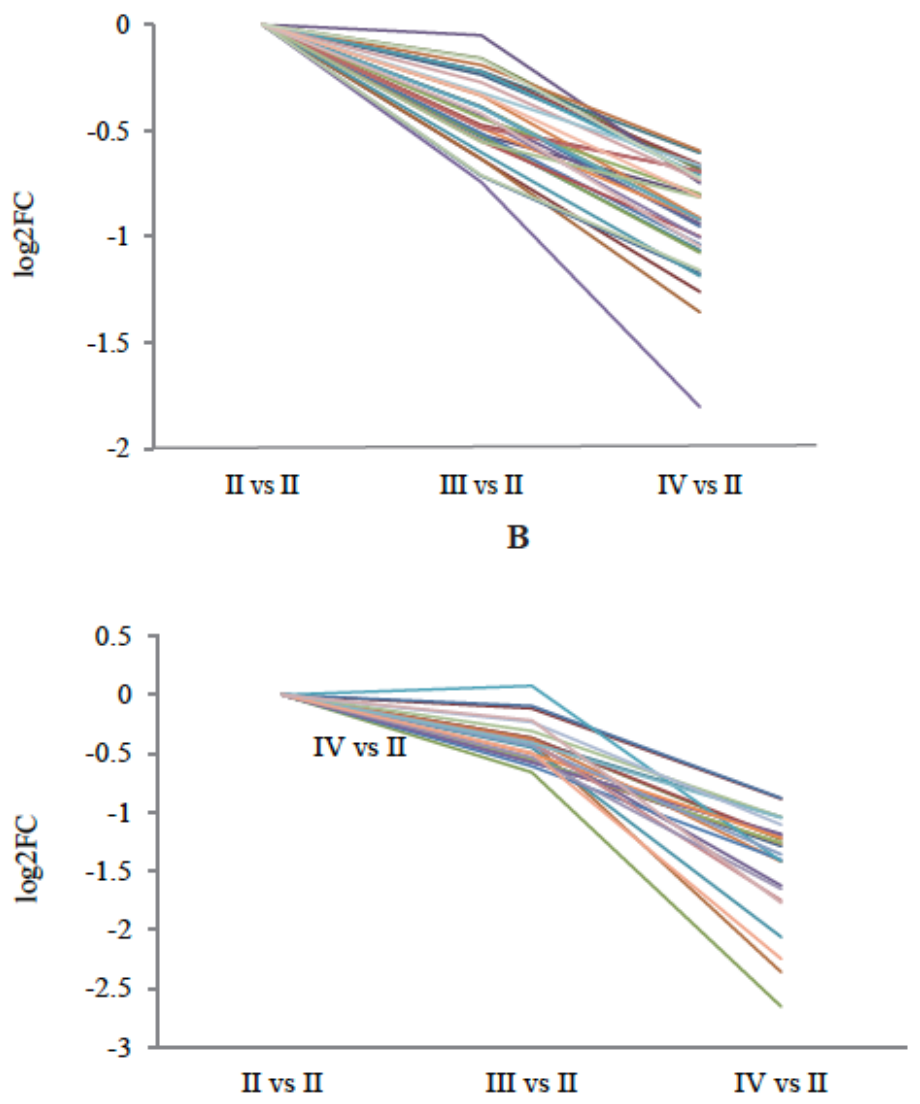

D

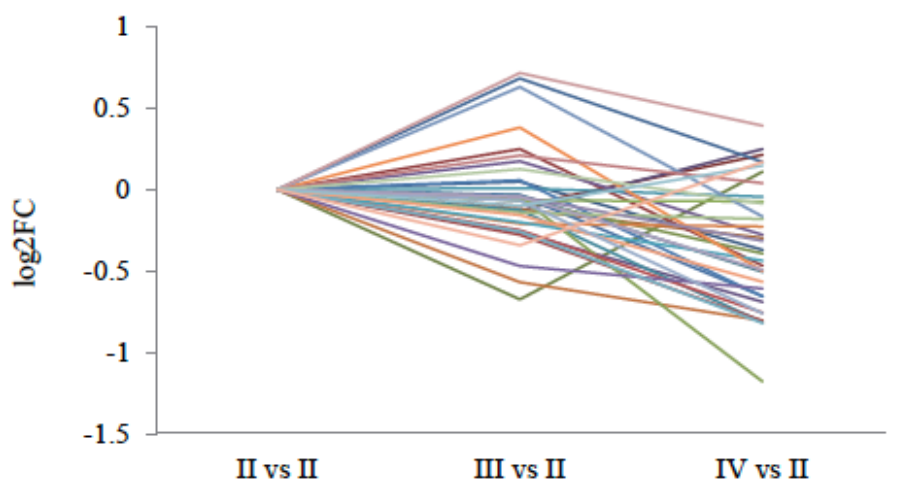

$\mathbf{F}$

\section{Figure 9}

The expression trend change of identified genes associated to development trchomes and roots. (A, C, E) Wild-type; (B, D, F) Mutant. 


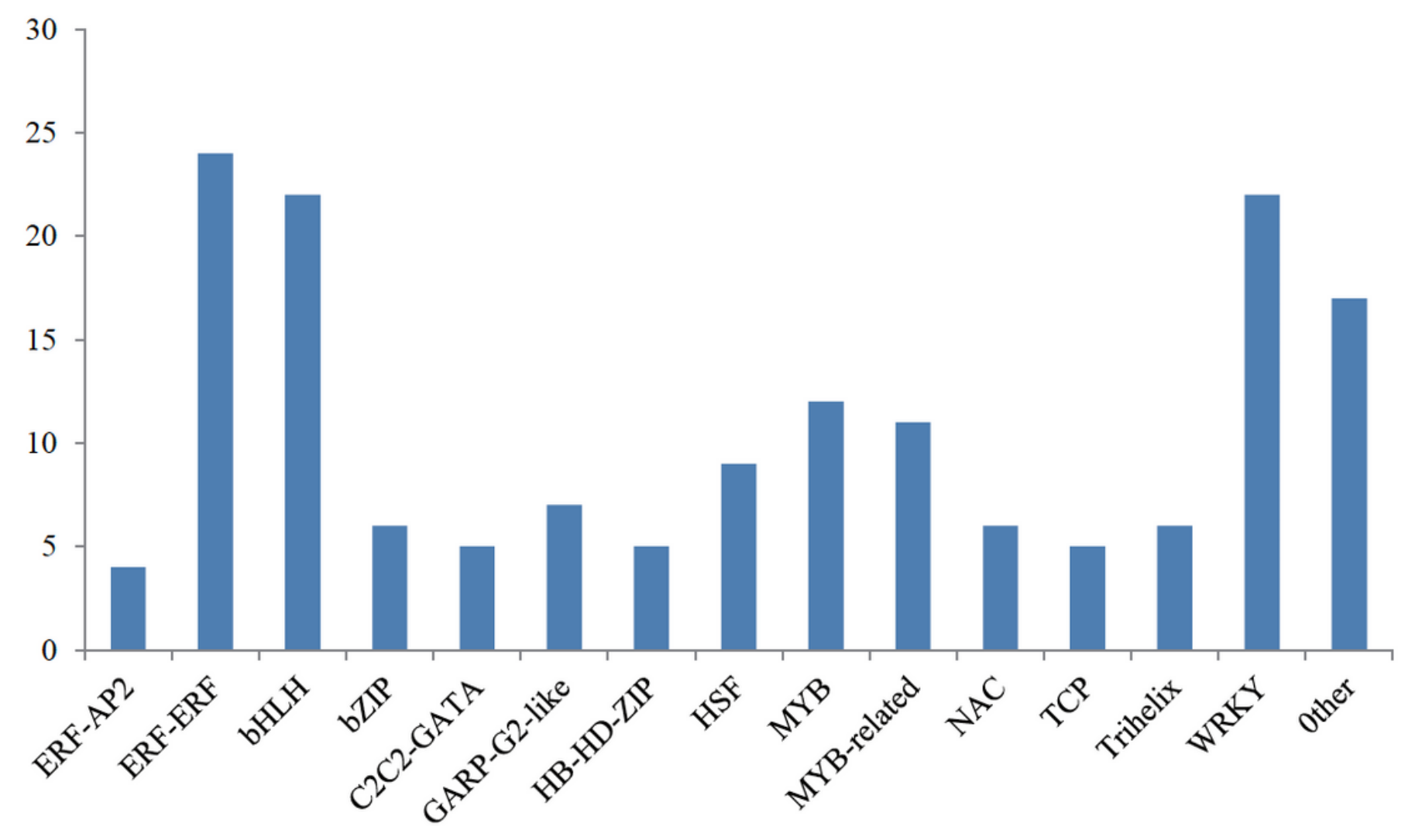

Figure 10

The distribution of transcription factor family from DEGs Other represents these families of genes with less than 3 members,include AP2/ERF-RAV, B3, BBR-BPC, BES1, C2C2-YABBY, C2H2, EIL, GeBP, GRAS, HBBELL, HB-other, HB-WOX, PLATZ, zf-HD

\section{Supplementary Files}

This is a list of supplementary files associated with this preprint. Click to download.

- supplement1.pdf

- supplement2.pdf

- supplement3.xIsx

- supplement4.xlsx

- supplement5.xlsx

- supplement6.xlsx

- supplement7.xlsx

- supplement8.xIsx

- supplement9.xIsx 
- supplement10.xlsx

- supplement11.xlsx

- supplement12.xlsx

- supplement13.xlsx

- supplement14.xlsx

- Table1.pdf

- Table2.xlsx

- Table3.xlsx 